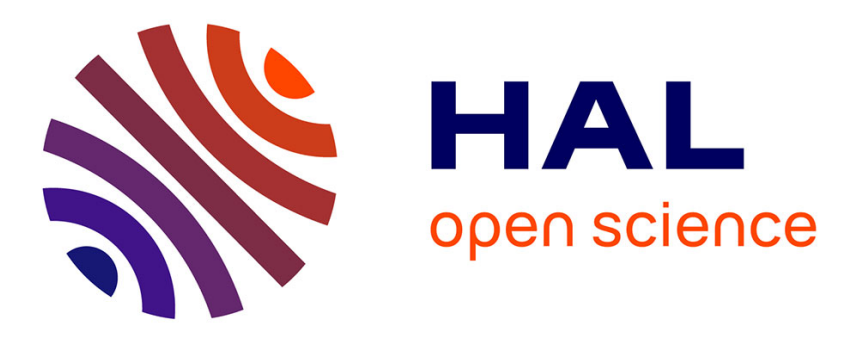

\title{
The ring finger protein 213 gene (Rnf213) contributes to Rift Valley fever resistance in mice
}

\author{
Denis Houzelstein, Dominique Simon-Chazottes, Leandro Batista, Satoko
} Tokuda, Francina Langa, Marie Flamand, Xavier Montagutelli, Jean-Jacques Panthier

\section{- To cite this version:}

Denis Houzelstein, Dominique Simon-Chazottes, Leandro Batista, Satoko Tokuda, Francina Langa, et al.. The ring finger protein 213 gene (Rnf213) contributes to Rift Valley fever resistance in mice. Mammalian Genome, 2021, 10.1007/s00335-020-09856-y · pasteur-03036189

\section{HAL Id: pasteur-03036189 \\ https://hal-pasteur.archives-ouvertes.fr/pasteur-03036189}

Submitted on 2 Dec 2020

HAL is a multi-disciplinary open access archive for the deposit and dissemination of scientific research documents, whether they are published or not. The documents may come from teaching and research institutions in France or abroad, or from public or private research centers.
L'archive ouverte pluridisciplinaire HAL, est destinée au dépôt et à la diffusion de documents scientifiques de niveau recherche, publiés ou non, émanant des établissements d'enseignement et de recherche français ou étrangers, des laboratoires publics ou privés.

\section{(ㅇ)(1) $\$$}

Distributed under a Creative Commons Attribution - NonCommerciall 4.0 International 


\section{The ring finger protein $\mathbf{2 1 3}$ gene (Rnf213) contributes to Rift}

\section{Valley fever resistance in mice}

Denis Houzelstein ${ }^{1,2,5^{*}}$, Dominique Simon-Chazottes ${ }^{1,2}$, Leandro Batista ${ }^{1,2}$, Satoko Tokuda $^{1,2}$, Francina Langa Vives ${ }^{3}$, Marie Flamand ${ }^{4}$, Xavier Montagutelli1 ${ }^{1,2,6,7}$ and Jean-Jacques Panthier ${ }^{1,2,7}$

1 Mouse Functional Genetics, Department of Developmental \& Stem Cell Biology, Institut Pasteur, Paris, 75015, France.

2 Centre National de la Recherche Scientifique, CNRS UMR3738, Paris, 75015, France.

${ }^{3}$ Mouse Genetics Engineering, Department of Developmental \& Stem Cell Biology, Institut Pasteur, Paris, 75015, France.

${ }^{4}$ Structural Virology, Department of Virology, Institut Pasteur, Paris, 75015, France.

5 Present address: Human Developmental Genetics, Department of Developmental \& Stem Cell Biology, Institut Pasteur, Paris, 75015, France.

${ }^{6}$ Mouse Genetics, Institut Pasteur, Paris, 75015, France.

7 These authors contributed equally.

*Corresponding author: denis.houzelstein@pasteur.fr

\section{ORCID number}

Denis Houzelstein 0000-0002-8536-9039

Xavier Montagutelli 0000-0002-9372-5398

Francina Langa Vives 0000-0002-0801-5464

Short Title: Rnf213 promotes resistance to Rift Valley fever 


\begin{abstract}
Rift Valley fever (RVF) is an emerging viral zoonosis that primarily affects ruminants and humans. We have previously shown that wild-derived MBT/Pas mice are highly susceptible to RVF virus and that part of this phenotype is controlled by a locus located on distal Chromosome 11. Using congenic strains, we narrowed down the critical interval to a $530 \mathrm{~kb}$ region containing five protein-coding genes among which Rnf213 emerged as a potential candidate. We generated Rnf213-deficient mice by CRISPR/CAS9 on the C57BL/6J background and showed that they were significantly more susceptible to RVF than control mice, with an average survival time post-infection reduced from 7 to 4 days. The human RNF213 gene had been associated with the cerebrovascular Moyamoya disease (MMD or MYMY) but the inactivation of this gene in the mouse resulted only in mild anomalies of the neovascularization. This study provides the first evidence that the Rnf213 gene may also impact the resistance to infectious diseases such as RVF.
\end{abstract}

Keywords: Rift Valley Fever virus, Rnf213, infectious disease 


\section{Introduction}

Rift Valley fever (RVF) is an emerging viral zoonosis caused by the RVF virus (RVFV) that primarily affects ruminants. A wide variation of symptoms is observed in infected animals, ranging from unapparent or moderate febrile reactions to high fever, severe prostration, and death in the most susceptible individuals (Ikegami and Makino 2011).

In humans, most RVF patients have no symptoms or present a self-limiting, febrile illness after a short incubation period of two to six days. Severe RVF disease accounts for 1-3\% of infected individuals. It manifests itself by acute-onset liver disease, neurological disorders, vision loss, or hemorrhagic fever with a fatality rate of $10-20 \%$ in hospitalized individuals (Ikegami and Makino 2011). Little is known, however, of the natural host factors that influence the progression and severity of RVF disease in animals and humans.

Evidence from experimental models has demonstrated the importance of genetic host factors in determining RVF disease severity in mice (do Valle et al. 2010; Smith et al. 2010). We have previously shown that individuals of the MBT/Pas (MBT) inbred strain, which derives from wild progenitors of the Mus musculus (M.m.) musculus subspecies, exhibit earlier lethality than mice of the BALB/cByJ (BALB/c) inbred strain due to defective activation of the innate immune response (AyariFakhfakh et al. 2012; do Valle et al. 2010). Quantitative trait locus (QTL) mapping identified three QTLs that contribute to RVF susceptibility in MBT mice (Tokuda et al. 2015). They were designated Rift Valley fever susceptibility 1 (Rvfs1), Rvfs2, and 
Rvfs3, and mapped on chromosome (Chr) 2, 11 and 5 respectively (Tokuda et al. 2015).

Differences in susceptibility to an infection may concern the mortality rate and/or the time to death, defined as the interval between infection and death in the mice succumbing to the infection. In our model of RVFV, we have often observed fluctuations in the mortality rate between experiments while the time to death has proven much more consistent. The Rvfs2 locus was identified as strongly influencing the time to death, which has become the focus of our studies. Moreover, we have reported that, in our model, males were significantly more susceptible than females (Tokuda et al. 2015), therefore our recent studies were done on male mice.

The characterization of the Rvfs2 locus was achieved using congenic mice. Indeed, while most BALB/c males die from 9 to 10 days after infection with signs of encephalitis (Batista et al. 2020), C.MBT-Rvfs2 congenic males, that carry the Rvfs2 locus from the MBT strain on a BALB/c genetic background, generally die within 5 days of infection with signs of acute hepatitis. We have shown that the prolonged time to death in BALB/c mice could be explained by their capacity to overcome this acute hepatitis, emphasizing the pathophysiological relevance of the time to death as a parameter for suceptibility (Batista et al. 2020).

In the present study, we have refined the genetic mapping of the Rvsf2 locus using overlapping subcongenic strains down to a 535-kb interval. Out of the five proteincoding genes contained in this critical interval, we identified Rnf213 as potentially interacting with several genes of the innate immune response. Rnf213 gene inactivation by a CRISP-Cas9-induced deletion resulted in a shorter survival time in 
Rnf213-deficient mice compared with wild-type or heterozygous mice demonstrating a role for Rnf213 in the resistance of this animal model to RVFV infection. 


\section{Results}

Generation, characterization and fine-mapping of subcongenic strains delimitate the susceptibility interval within $535 \mathrm{~kb}$

The Rvfs2 QTL, which controls the average time to death in RVFV-lethally infected mice, was previously isolated in the C.MBT-Rvfs 2 congenic strain which carries a segment of $\sim 16.9 \mathrm{Mb}$ on distal Chr 11 (Tokuda et al. 2015). This strain now renamed C.MBT-Rvfs2-1 was crossed with BALB/c mice to produce a series of subcongenic strains numbered C.MBT-Rvfs2-2 (Rvfs2-2) to C.MBT-Rvfs2-8 (Rvfs2-8) and shown in Figure 1A (see Table S1 for the list of markers used to map the recombination sites in the sub-congenic strains). Groups of homozygous subcongenic males were tested for their susceptibility to RVFV infection (Figure 1B). As previously observed, the average time to death in lethally-infected C.MBT-Rvfs2-1 and BALB/c mice was 4.13 days and 7.38 days, respectively (Table 1). It appeared that the subcongenic strains could be sorted into two groups according to the average time to death : Rvfs2-3 and Rvfs2-7 (average time to death 8.06 and 6.78 days, respectively, as long as BALB/c mice), and all the other strains, namely Rvfs2-2, Rvfs 2-4, Rvfs2-5, Rvfs2-6, and Rvfs2-8, in which susceptible mice died in less than 4.8 days, close to the $R v f s 2-1$ congenic strain. Time to death was significantly longer in strains of the first group compared with the second group (Table 1) This result allowed us to narrow down the genomic interval containing the BALB/c resistance allele(s) to the interval spanning $535 \mathrm{~kb}$ between positions 119.393 and $119.928 \mathrm{Mb}$ according to GRCm38-mm10 (Table S1). Analysis of the $535 \mathrm{~kb}$ susceptibility interval identified five protein-coding genes of known function (Rnf213, EndoV, Nptx1, Rptor and Chmp6), four long noncoding RNAs (A930037H05Rik, Gm39484, Gm11762 and Gm39485), one antisense 
long non-coding RNA (Rptoros) and one predicted sno RNA gene (Gm23663). To prioritize the genes most likely to be responsible for the phenotype of Rvfs2-1 mice, we focused on protein-coding genes and used the STRING Mus musculus database to identify their interaction networks (Snel et al. 2000; Szklarczyk et al. 2015). This analysis, performed in April 2017, revealed that mouse RNF213 formed a highly interconnected network with UBA7, USP18, PARP14, IFIT3, IRF7, RSAD2, STAT1, IGTP, MX2, RTP4, OASL2, IRGM2, LGALS3BP, and OAS2 proteins (Figure S1). Seven of these 14 proteins were associated with the Gene Ontology (G0) categories 'innate immune response' (G0:0045087), 6 with 'immune effector process' (G0:0002252) and/or 'response to other organism' (G0:0051707), 5 with 'defense response to virus' (GO:0051607), 3 with 'negative regulation of viral process' (GO:0048525), and 2 with 'response to type I interferon' (G0:0034340). Considering its numerous interactions with innate immunity genes, we prioritized $R n f 213$ for candidate gene studies.

\section{Rnf213-deficient mice are more susceptible to RVFV infection}

The mouse Rnf213 gene encodes a 591-kDa cytosolic protein that possesses two main functional domains that we located by homology with other mammalian sequences (Figure 2A). The first domain (amino acids 2330 to 2964 of the mouse protein) is formed by a pair of AAA+ (ATPases associated with diverse cellular activities). ATPase modules are defined by specific structures surrounding Walker A and B motifs (Morito et al. 2014). It is encoded by exon 29 (Figures 2B and C). The second domain (amino acids 3877 to 3984) is a RING-finger-containing E3 ubiquitin ligase, PEX10 (COG5574) domain. It is encoded by exons 41 to 46 (Figure 2B). 
Since the genome of the Mus musculus musculus-derived MBT/Pas strain has not been sequenced, we considered the $\mathrm{PWK} / \mathrm{PhJ}$ strain, which belongs to the same subspecies, as a proxy for MBT/Pas. The sequence comparison between PWK/Pas and BALB/c strains (using the Sanger Institute's Mouse Genomes Project) identified 51 non-synonymous SNPs and 4 indels dispersed over most domains of the protein (Table S2). Eleven of these SNPs are located in splicing regions. Although variants between MBT/Pas and BALB/c may only partially overlap this set, these variants are too many to predict their cumulative functional impact (expression level, protein structure, stability or function).To assess the role of the Rnf213 gene in RVFV infection, we produced Rnf213-deficient mice (referred to as $R n f 213^{\mathrm{tm} 3 / \mathrm{tm} 3}$ ) by deleting the $\mathrm{AAA}+$ domain encoding sequence (Figure 2C) and generating a frameshift by subsequent repair using non-homologous end joining (NHEJ, Figure 2C). The resulting truncated protein is predicted to contain neither of the two RNF213 characteristic conserved domains (Figure 2D).

Out of the founders obtained, two (\#4 and 17) presented the expected truncation as revealed by sequencing and were used for strain establishment. Homozygous mutants did not show any visible phenotype under conventional environment as reported on previous $R n f 213^{t m / t m}$ mouse strains (Kobayashi et al. 2015; Sonobe et al. 2014).

After intra-peritoneal injection of $10^{2}$ plaque-forming units (PFU) of the RVFV strain ZH548, about $13 \%$ of the C57BL/6J mice infected with RVFV survived the infection (2/15, Figure 3$)$, not significantly different from that of $\operatorname{Rnf} 213^{\mathrm{tm} 3 / \mathrm{tm} 3}(1 / 28=3.6 \%)$ and $R n f 213^{\text {tm } 3 /+}(0 / 10)$ mice $(\mathrm{p}=0.27$ and $\mathrm{p}=0.5$, respectively, Fisher's exact test). 
However, the survival time in lethally infected mice was significantly shorter in $R n f 213^{t m 3 / t m 3}(4.93 \pm 0.28$ days, mean \pm s.e.m. $)$ than in $R n f 213^{+/+}(7.15 \pm 0.45$ days,

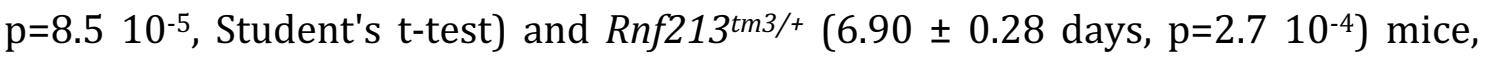
resulting in highly significant differences in survival curves (Figure ). Similar data were obtained with mutant mice of both \#4 and 17 strains. These results indicate that the Rnf213 gene delays the fatal outcome of RVFV infection in the C57BL/6J genetic background and that one functional allele is sufficient to confer this phenotype.

\section{Discussion}

Several studies by us and others have demonstrated that the outcome of RVFV infection is strongly influenced by multiple host genes. In particular, we have reported three RVF susceptibility loci affecting the time to death in susceptible mice (Tokuda et al. 2015). The Rvfs2 locus was previously isolated in a congenic strain carrying a 16.9 Mb segment of distal Chr 11 of the highy susceptible MBT strain in a BALB/c inbred background. We have previously shown that these congenic mice die earlier than BALB/c mice due to a higher susceptibility to the acute RVFVinduced hepatitis correlated with increased replication rate in hepatocytes (Batista et al. 2020). This result provided some functional evidence for the specific effect of the Rvfs2 locus on the time to death in mice which develop a fatal infection.

In the present study, we aimed at identifying the genetic determinants responsible for the effect of $R v f s 2$. Genetic dissection of a large locus using subcongenic strains is a tedious process but also a rigourous approach for narrowing the position of causal variants. In our case, it allowed us to reduce the genomic interval down to 
$535 \mathrm{~kb}$ containing only 5 protein-coding genes, none of which had been so far involved in host genetic control of susceptibility to infections. We selected Rnf213 as a candidate based on its interaction with several genes involved in the innate immune response. The production of a loss of function variant allowed us to assess the importance of Rnf213 in the susceptibility to RVFV in mice.

Since the genome of the MBT strain has not been sequenced, we lack a comprehensive inventory of the variants between the MBT and BALB/c alleles of the Rnf213 gene. However, the comparison between $\mathrm{PWK} / \mathrm{PhJ}$, a related inbred strain of the same mouse subspecies as MBT, and BALB/c revealed a large number of variants which could possibly alter the expression, protein structure or functional activity. Whether one or several of these genetic variants is responsible for the susceptibility to RVFV-induced acute hepatitis conferred by the Rvfs2 locus remains to be determined. However, this hypothesis is reasonable since Rnf213-deficient mice show similar decrease of their survival time as C.MBT-Rvfs2 mice.

Researchers have shown little interest in the human RNF213 gene until 2011 when two groups independently demonstrated that SNPs in the gene were strongly associated with the onset of the cerebrovascular Moyamoya disease (MMD or MYMY) in both familial and sporadic cases ((Kamada et al. 2011; Liu et al. 2011), see (Koizumi et al. 2016; Lin and Sheng 2018) for reviews). Several variants of RNF213 induce MYMY (MYMY2, OMIM \#607151) with no other recognizable phenotypes. The disease was first described in Japan in 1957. It is a steno-occlusive disease of the cerebral arteries, involving smooth muscle cell proliferation with intima hyperplasia causing arterial stenosis and occlusion around the circle of Willis. This, 
in turn, stimulates the compensatory development of collateral vessels, which have a "puff of smoke" (Moyamoya in Japanese) appearance in cerebral angiography (Koizumi et al. 2016; Lin and Sheng 2018). The mechanism by which RNF213 variants relate to MYMY remains unknown. Knockdown of RNF213 in cultured endothelial cells results in less angiogenic profiles suggesting that RNF213 and its upstream pathways contribute to the process of angiogenesis (Ohkubo et al. 2015). Its expression is activated by inflammatory signals from the environment both in vivo and in vitro. Okhubo and colleagues therefore proposed that RNF213 links inflammatory and angiogenic signals in endothelial cells (Ohkubo et al. 2015). The most frequent MYMY causing variant, R4810K, frequent in East Asian populations, has also been strongly associated with hypertension (Koizumi et al. 2013). At this stage, it is still difficult to link a role for Rnf213 in the response to RVFV infection in mice and the documented role for RNF213 in hypertension and angiopathies in humans.

RNF213 is an intracellular protein that forms large oligomers (Morito et al. 2014). It exerts ubiquitylation activity toward a variety of substrate proteins including itself (Kotani et al. 2017; Liu et al. 2011). RNF213 is targeted to lipid droplets, ubiquitous organelles specialized for neutral lipid storage, and markedly increases their abundance in cells (Sugihara et al. 2019). Depletion of RNF213 actually protects cells from palmitate-mediated cellular toxicity and normalizes the palmitate-induced changes in gene expression (Piccolis et al. 2019).

There are several stages during the course of RVFV infection where cellular lipids derived from de novo palmitate production are utilized. Reducing levels of fatty acid synthesis restricts RVFV infection in cell cultures. In contrast, restoring the level of 
fatty acids by exogenous addition of palmitate, the first product of fatty acid biosynthesis, restores infection (Moser et al. 2012). Dependence on lipid biosynthesis and virally induced membrane modifications is not unique to RVFV; many RNA viruses require extensive membrane modifications and proliferations to support their replication complex (Filipe and McLauchlan 2015; Moser et al. 2012; Ogawa et al. 2009).

In human RVFV patients, the liver is the primary site of RVFV replication and lipid droplet accumulation was reported in the liver of a man who died from RVFV (Shraim et al. 2016). In the mouse model, a major consequence of RVFV infection is also the overwhelming infection of hepatocytes that subsequently undergo apoptosis resulting in early-onset death primarily attributed to severe hepatitis (Batista et al. 2020; Smith et al. 2010). The hepatocytes that maintain their viability amass cytoplasmic lipid droplets that persist during liver regeneration in the surviving mice (Reed et al. 2012). It is therefore possible that Rnf213 influences resistance to RVFV infection through a role in lipid metabolism.

In conclusion, we have identified a new function for the Rnf213 gene as a host factor controlling resistance to RVFV infections in mice. Review of the current literature suggests its effect may be mediated by a role in the lipid metabolism. It will therefore be of interest to determine whether Rnf213 affects the infection outcome of other viruses in mouse models and whether $R n f 213$ and its variants have an impact on susceptibility to viral infections in human as well. 


\section{Materials and Methods}

\section{Ethics statement}

Experiments on mice were conducted according to the French and European regulations on care and protection of laboratory animals (EC Directive 2010/63/UE and French Law 2013-118 issued on February 1, 2013). The protocols were approved by the Animal Ethics Committees of Paris Center and South ( $\left.\mathrm{N}^{\circ} 59\right)$ and of the Institut Pasteur ( $\mathrm{N}^{\circ}$ 89) and authorized by the French Ministry of Research (under references \#06463 and 14646).

Mice

C57BL/6J mice were purchased from Janvier Labs (Le Genest-Saint-Isle, France). BALB/cByJ mice were purchased from Charles River Laboratories France (L'Arbresle, France). Subcongenic mice were obtained by backcrossing C.MBTRvfs2-1 mice (similar to C.MBT-Rvfs2 described by Tokuda et al. (Tokuda et al. 2015)) with BALB/cByJ mice and by genotyping progeny using polymorphic microsatellite or SNP markers. Several generations of backcrosses were necessary to obtain the recombination breakpoints present in C.MBT-Rvfs2-2 to C.MBT-Rvfs28 congenic strains.

\section{Virus production and mouse infection}

The RVFV strain ZH548, isolated from a male patient with the acute febrile illness at Zagazig fever hospital, Egypt (El-Akkad 1978; Meegan 1979), obtained from Centre National de Référence des Fièvres Hémorragiques Virales, Institut Pasteur, Lyon, France, was used for all infection studies. All experiments that involved virulent RVFV were performed in the biosafety level 3 (BSL3) facilities of the Institut Pasteur, and carried out in compliance with the recommendations of the Institut Pasteur 
Biosafety Committee $\left(\mathrm{N}^{\circ}\right.$ 14.320). Stocks of RVFV ZH548 were titrated by plaque assay on monolayers of Vero E6 cells (Billecocq et al. 2008). Nine to thirteen weeks old male mice were infected by intraperitoneal (i.p.) injection of $10^{2} \mathrm{PFU}$ RVFV. Morbidity and mortality were monitored daily for 11 to 14 days following infection.

\section{Generation of CRISPR/Cas9 mutants}

Single guide RNAs (sgRNAs) were prepared according to (Cong et al. 2013), as described by Raveux et al. (Raveux et al. 2017). For each guide sequence, an oligonucleotide pair was annealed and cloned into a BbsI-digested pX458 expression vector. After sequencing, PCR products were used as templates with MEGAshortscript T7 and mMESSAGE mMACHINE T7 transcription kits (Life Technologies, Carlsbad, CA, USA) for the guides and Cas9, respectively. sgRNAs and Cas9 mRNA were then precipitated with $\mathrm{LiCl} /$ ethanol and resuspended in Brinster's buffer (10 mM Tris-HCl pH 7.5, 0.25 mM EDTA).

To increase the probability of obtaining non-homologous end joining events, we designed 4 guides in the exon 29 of Rnf213 which encodes the tandem AAA+ ATPase domains. Two guides target sequences $5^{\prime}$ to the AAA+ ATPase domains $\left(5^{\prime}-1\right.$ : TTAAATACTGGTAAGGTCGTTGG and 5'-2: CACTTCCTTGGGAGGACGGCAGG), whereas the remaining two guides target sequences 3' to the AAA+ATPase domains (3'-1: TGTGCCCCTCATCAACCGTCTGG and 3'-2: CTCGATGACGATGAGGCGGAAGG). The guides (50ng/ $\mu \mathrm{l}$ each) and Cas9 mRNA (100ng/ $\mu \mathrm{l})$ were microinjected into C57BL/6J one-cell-stage embryos. The resulting pups were screened by PCR with three primers (rnf213-diag5L-1f: TGTGTTAGAAAATGCATCTTTGTTAGGG, rnf213diag3C-1f: TGCTCTCAACCAGTACTATGTCTACCTTGG, and rnf213-diag3C-1r: CAATGAACTGGTCTGCTTTTACATCTGCG). The predicted PCR products of the wild- 
type and mutant alleles were 281 bp (rnf213-diag5L-1f_rnf213-diag3C-1r) and 450bp (rnf213-diag3C-1f_ rnf213-diag3C-1r) in length, respectively (Figure 2C). Exon 29 was sequenced in several mutants to confirm the predicted deletion (Figure 2D). The sequence of the RNF213 ${ }^{\mathrm{tm} 3}$ proteins is shown in Figure 2E.

\section{Statistical analysis}

Gehan-Breslow-Wilcoxon test for survival analysis and Fisher's exact test were performed using GraphPad Prism version 8.4.1 for MacOS (GraphPad Software, La Jolla California USA). 


\section{DATA AVAILABILITY STATEMENT}

All relevant data are within the article

\section{CONFLICT OF INTEREST}

The authors declare no conflict of interest.

\section{ACKNOWLEDGMENTS}

We are grateful to all members of the laboratory for technical advice and helpful discussion. We are grateful to Lauranne Machou and María Bernad Roche for their precious help during their short stay in the laboratory. . We would like to thank the members of the Mouse Genetics Engineering Center, Sébastien Chardenoux, Ilta Lafosse, and Gaëlle Chauveau Le Friec for technical support with transgenic mice. This work was supported by the Agence Nationale de la Recherche (grant $\mathrm{n}^{\circ} 11$ BSV3-007 01, 'GenRift') and the French Government's Investissement d'Avenir program, Laboratoire d'Excellence Integrative Biology of Emerging Infectious Diseases (grant nºAR-10-LABX-62-IBEID).

\section{AUTHOR CONTRIBUTION}

Denis Houzelstein conceived the study, performed the experiments, analysed the data, and wrote the manuscript.

Dominique Simon-Chazottes performed in vivo experiments.

Leandro Batista and Satoko Tokuda produced congenic strains.

Francina Langa Vives and her team produced the Rnf213 ${ }^{\text {tm } 3}$ founder individuals. Marie Flamand and her team assisted with RVFV production and titration. Xavier Montagutelli assisted with data analysis, performed in vivo experiments, and wrote the manuscript.

Jean-Jacques Panthier provided funding, conceived the study, and wrote the manuscript. 


\section{FIGURE LEGENDS}

\section{Figure 1.}

A. Schematic representation of the genomic intervals harbored by the congenic $R v f s 2-1$ and the subcongenic $R v f s 2-2$ to $R v f s 2-8$ strains produced to fine map the Rvfs2 QTL. The black bars represent the regions homozygous for the BALB/c genome (the background strain) and the grey bars represent the regions homozygous for the donor MBT genome. E: eary lethality (similar to Rvfs2.1); L: late lethality (similar to BALB/c). On the right are shown the approximate positions of the boundaries of the minimal interval and the genes it contains.

B. Survival curves of C.MBT-Rvfs2 congenic and subcongenic (Rvfs2-1 to Rvfs2-8) and BALB/c mice after infection with 100 PFU of RVFV ZH548 strain. Numbers of mice are given in brackets.

\section{Figure 2.}

Structure of the Rnf213 gene and the CRISP-Cas9-induced mutation. A: Organization of the RNF213 protein. The position of the RNF213 conserved domains is indicated. B: Structure of the endogenous mouse Rnf213 gene highlighting the position of exon 29 which encodes the AAA+ domains. C: The guides used to generate the deleted allele are shown as black arrows relative to the AAA+ domains. The primers used for genotyping transgenic mice are shown in red with the expected amplicon size. The expected deletion is shown as a triangle. PCR genotyping of Rnf213+/, $R n f 213^{t m 3 / t m 3}(\Delta / \Delta)$ and $R n f 213^{+/ t m 3}$ mice using primers: diag5L-1f, diag3C-1F and diag3C-1r . D: Genomic deletion in the two $R n f 213^{\text {tm } 3}$ alleles analyzed. Rnf213 2 tm3-17 
carries a 6 base pair insertion within the deletion. E: Amino acid sequence of the C57BL/6J Rnf213 protein and of the truncated protein predicted from the deleted nucleotide sequence of the $R n f 213^{t m 3 / t m 3}-4$ and $R n f 213^{t m 3 / t m 3-17}$ mutants.

\section{Figure 3.}

Survival curves of $R n f 213^{t m 3 / t m 3}, R n f 213^{t m 3 /+}$, and C57BL/6J $\left(R n f 213^{+/+}\right)$male mice after infection with 100 PFU of RVFV ZH548 strain. Numbers of mice are given in brackets. Statistical test: Gehan-Breslow-Wilcoxon test, ${ }^{* * *(1)} \mathrm{p}=0.0002,{ }^{* * *(2)}$ $\mathrm{p}=0,0010$.

\section{Table 1.}

$\S$ comparison of the survival time between the first group of strains (BALB/cByJ, C.MBT-Rvfs2-3 and C.MBT-Rvfs2-7) and all the others; strains of the second group did not statistically differ between them; numbers refer to C.MBT-Rvfs2 substrains; $* *: \mathrm{p}<0.01 ; * * * \mathrm{p}<0.001 ;{ }^{* * * *}: \mathrm{p}<0.0001$. 


\section{References}

Ayari-Fakhfakh E, do Valle TZ, Guillemot L, Panthier JJ, Bouloy M, Ghram A, Albina E, CetreSossah C (2012) MBT/Pas mouse: a relevant model for the evaluation of Rift Valley fever vaccines. The Journal of general virology 93, 1456-1464

Batista L, Jouvion G, Simon-Chazottes D, Houzelstein D, Burlen-Defranoux O, Boissière M, Tokuda S, do Valle TZ, Cumano A, Flamand M, Montagutelli X, Panthier J-J (2020) Genetic dissection of Rift Valley fever pathogenesis: Rvfs2 locus on mouse chromosome 11 enables survival to early-onset hepatitis. Scientific Reports 10

Billecocq A, Gauliard N, Le May N, Elliott RM, Flick R, Bouloy M (2008) RNA polymerase Imediated expression of viral RNA for the rescue of infectious virulent and avirulent Rift Valley fever viruses. Virology 378, 377-384

Cong L, Ran FA, Cox D, Lin SL, Barretto R, Habib N, Hsu PD, Wu XB, Jiang WY, Marraffini LA, Zhang F (2013) Multiplex Genome Engineering Using CRISPR/Cas Systems. Science $339,819-823$

do Valle TZ, Billecocq A, Guillemot L, Alberts R, Gommet C, Geffers R, Calabrese K, Schughart K, Bouloy M, Montagutelli X, Panthier JJ (2010) A New Mouse Model Reveals a Critical Role for Host Innate Immunity in Resistance to Rift Valley Fever. The Journal of Immunology 185, 6146-6156

El-Akkad AM (1978) Rift Valley fever outbreak in Egypt. October--December 1977. The Journal of the Egyptian Public Health Association 53, 123-128

Filipe A, McLauchlan J (2015) Hepatitis C virus and lipid droplets: finding a niche. Trends Mol Med 21, 34-42

Ikegami T, Makino S (2011) The Pathogenesis of Rift Valley Fever. Viruses 3, 493-519

Kamada F, Aoki Y, Narisawa A, Abe Y, Komatsuzaki S, Kikuchi A, Kanno J, Niihori T, Ono M, Ishii N, others (2011) A genome-wide association study identifies RNF213 as the first Moyamoya disease gene. Journal of human genetics 56, 34-40

Kobayashi H, Matsuda Y, Hitomi T, Okuda H, Shioi H, Matsuda T, Imai H, Sone M, Taura D, Harada KH, Habu T, Takagi Y, Miyamoto S, Koizumi A (2015) Biochemical and Functional Characterization of RNF213 (Mysterin) R4810K, a Susceptibility Mutation of Moyamoya Disease, in Angiogenesis In Vitro and In Vivo. Journal of the American Heart Association: Cardiovascular and Cerebrovascular Disease 4, e002146

Koizumi A, Kobayashi H, Hitomi T, Harada KH, Habu T, Youssefian S (2016) A new horizon of moyamoya disease and associated health risks explored through RNF213. Environmental Health and Preventive Medicine 21, 55-70

Koizumi A, Kobayashi H, Liu W, Fujii Y, Senevirathna ST, Nanayakkara S, Okuda H, Hitomi T, Harada KH, Takenaka K, Watanabe T, Shimbo S (2013) P.R4810K, a polymorphism of RNF213, the susceptibility gene for moyamoya disease, is associated with blood pressure. Environ Health Prev Med 18, 121-129

Kotani Y, Morito D, Sakata K, Ainuki S, Sugihara M, Hatta T, Iemura S-i, Takashima S, Natsume T, Nagata K (2017) Alternative exon skipping biases substrate preference of the deubiquitylase USP15 for mysterin/RNF213, the moyamoya disease susceptibility factor. Scientific Reports 7, 44293

Lin J, Sheng W (2018) RNF213 Variant Diversity Predisposes Distinct Populations to Dissimilar Cerebrovascular Diseases. Biomed Res Int 2018, 6359174

Liu W, Morito D, Takashima S, Mineharu Y, Kobayashi H, Hitomi T, Hashikata H, Matsuura N, Yamazaki S, Toyoda A, Kikuta K-i, Takagi Y, Harada KH, Fujiyama A, Herzig R, Krischek B, Zou L, Kim JE, Kitakaze M, Miyamoto S, Nagata K, Hashimoto N, Koizumi A (2011) 
Identification of RNF213 as a Susceptibility Gene for Moyamoya Disease and Its Possible Role in Vascular Development. PLoS ONE 6, e22542

Meegan JM (1979) The Rift Valley fever epizootic in Egypt 1977-78. 1. Description of the epizzotic and virological studies. Transactions of the Royal Society of Tropical Medicine and Hygiene 73, 618-623

Morito D, Nishikawa K, Hoseki J, Kitamura A, Kotani Y, Kiso K, Kinjo M, Fujiyoshi Y, Nagata K (2014) Moyamoya disease-associated protein mysterin/RNF213 is a novel AAA+ ATPase, which dynamically changes its oligomeric state. Scientific Reports 4

Moser TS, Schieffer D, Cherry S (2012) AMP-Activated Kinase Restricts Rift Valley Fever Virus Infection by Inhibiting Fatty Acid Synthesis. PLoS Pathogens 8, e1002661

Ogawa K, Hishiki T, Shimizu Y, Funami K, Sugiyama K, Miyanari Y, Shimotohno K (2009) Hepatitis $\mathrm{C}$ virus utilizes lipid droplet for production of infectious virus. Proc Jpn Acad Ser B Phys Biol Sci 85, 217-228

Ohkubo K, Sakai Y, Inoue H, Akamine S, Ishizaki Y, Matsushita Y, Sanefuji M, Torisu H, Ihara K, Sardiello M, Hara T (2015) Moyamoya disease susceptibility gene RNF213 links inflammatory and angiogenic signals in endothelial cells. Scientific Reports 5, 13191

Piccolis M, Bond LM, Kampmann M, Pulimeno P, Chitraju C, Jayson CBK, Vaites LP, Boland S, Lai ZW, Gabriel KR, Elliott SD, Paulo JA, Harper JW, Weissman JS, Walther TC, Farese RV, Jr. (2019) Probing the Global Cellular Responses to Lipotoxicity Caused by Saturated Fatty Acids. Mol Cell

Raveux A, Vandormael-Pournin S, Cohen-Tannoudji M (2017) Optimization of the production of knock-in alleles by CRISPR/Cas9 microinjection into the mouse zygote. Sci Rep 7, 42661

Reed C, Steele KE, Honko A, Shamblin J, Hensley LE, Smith DR (2012) Ultrastructural study of Rift Valley fever virus in the mouse model. Virology 431, 58-70

Shraim MA, Eid R, Radad K, Saeed N (2016) Ultrastructural pathology of human liver in Rift Valley fever. BMJ Case Rep 2016

Smith DR, Steele KE, Shamblin J, Honko A, Johnson J, Reed C, Kennedy M, Chapman JL, Hensley LE (2010) The pathogenesis of Rift Valley fever virus in the mouse model. Virology 407, 256-267

Snel B, Lehmann G, Bork P, Huynen MA (2000) STRING: a web-server to retrieve and display the repeatedly occurring neighbourhood of a gene. Nucleic acids research 28 , 3442-3444

Sonobe S, Fujimura M, Niizuma K, Nishijima Y, Ito A, Shimizu H, Kikuchi A, Arai-Ichinoi N, Kure S, Tominaga T (2014) Temporal profile of the vascular anatomy evaluated by 9.4-T magnetic resonance angiography and histopathological analysis in mice lacking RNF213: A susceptibility gene for moyamoya disease. Brain Research 1552, 64-71

Sugihara M, Morito D, Ainuki S, Hirano Y, Ogino K, Kitamura A, Hirata H, Nagata K (2019) The AAA+ ATPase/ubiquitin ligase mysterin stabilizes cytoplasmic lipid droplets. J Cell Biol 218, 949-960

Szklarczyk D, Franceschini A, Wyder S, Forslund K, Heller D, Huerta-Cepas J, Simonovic M, Roth A, Santos A, Tsafou KP, Kuhn M, Bork P, Jensen LJ, von Mering C (2015) STRING v10: protein-protein interaction networks, integrated over the tree of life. Nucleic acids research 43, D447-452

Tokuda S, Do Valle TZ, Batista L, Simon-Chazottes D, Guillemot L, Bouloy M, Flamand M, Montagutelli X, Panthier JJ (2015) The genetic basis for susceptibility to Rift Valley fever disease in MBT/Pas mice. Genes and Immunity 16, 206-212 


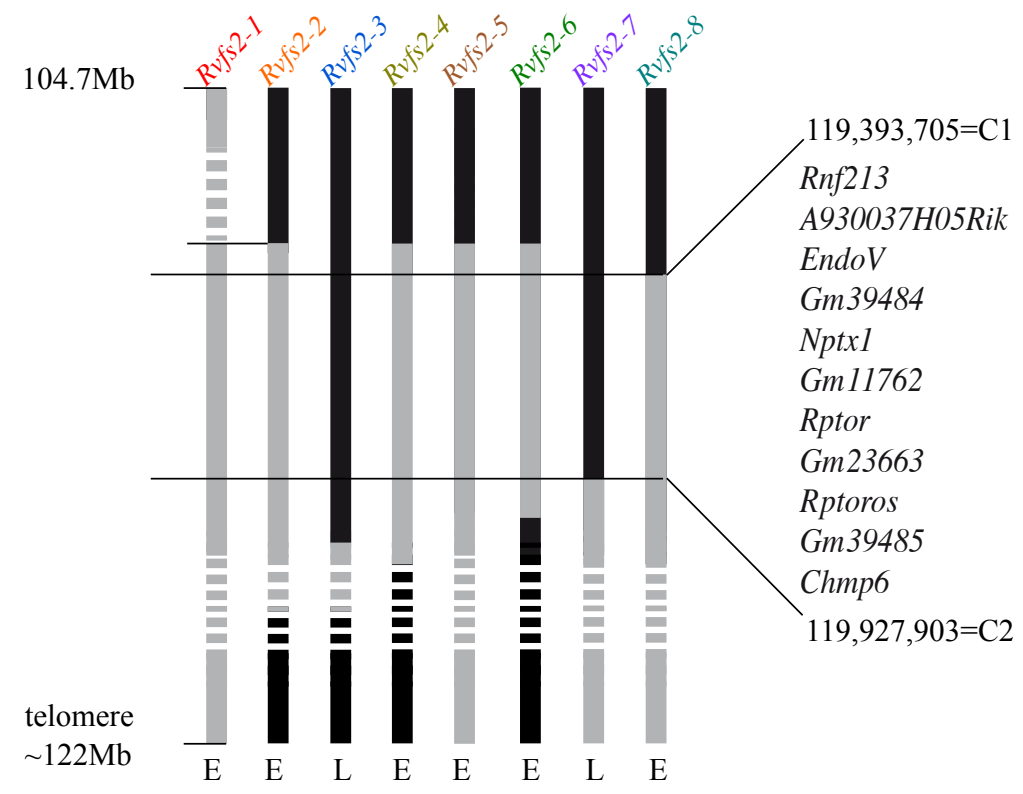

B

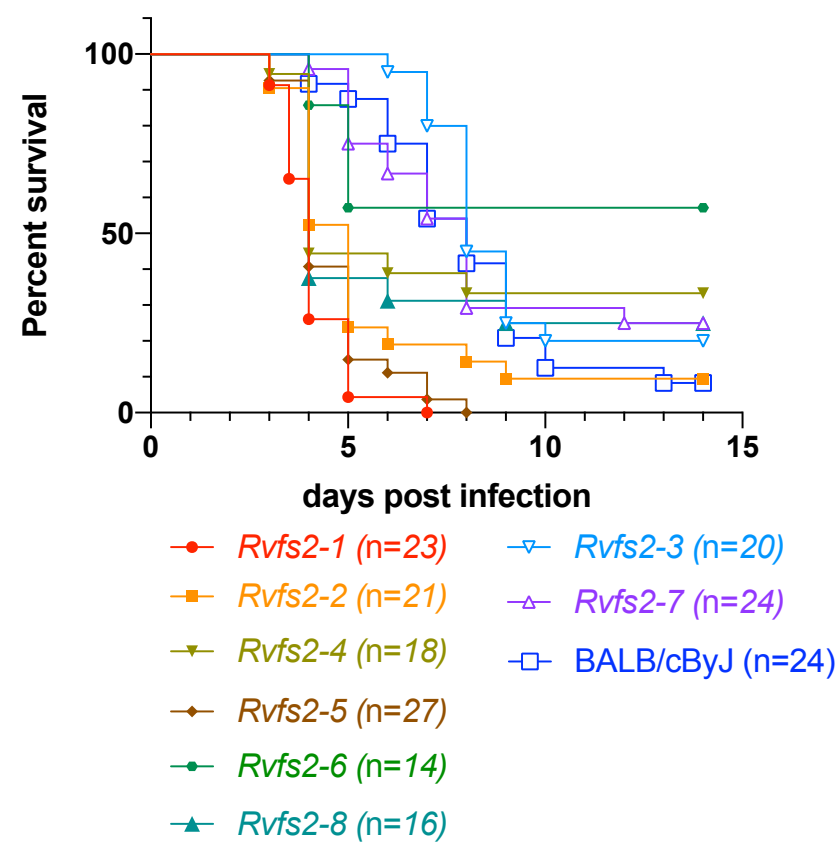

\section{Figure 1.}

A. Schematic representation of the genomic intervals harbored by the congenic $R v f s 2-1$ and the subcongenic $R v f s 2-2$ to $R v f s 2-8$ strains produced to fine map the $R v f s 2$ QTL. The black bars represent the regions homozygous for the $\mathrm{BALB} / \mathrm{c}$ genome (the background strain) and the grey bars represent the regions homozygous for the donor MBT genome. E: eary lethality (similar to Rvfs2.1); L: late lethality (similar to BALB/c). On the right are shown the approximate positions of the boundaries of the minimal interval and the genes it contains.

B. Survival curves of C.MBT-Rvfs2 congenic and subcongenic (Rvfs2-1 to $R v f s 2-8)$ and BALB/c mice after infection with 100 PFU of RVFV ZH548 strain. Numbers of mice are given in brackets. 
Table 1 : Survival time in lethally-infected mice

\begin{tabular}{lcc}
\hline Mouse strain & $\begin{array}{c}\text { Survival time in days } \\
\text { mean } \pm \text { s.e.m. }\end{array}$ & $\begin{array}{c}\text { Comparisons } \\
\text { (Mann-Whitney) }\end{array}$ \\
\hline BALB/cByJ & $7.38 \pm 0.39$ & $\begin{array}{c}1,2,4,5,8:^{* * * *} \\
6::^{* *}\end{array}$ \\
\hline C.MBT-Rvfs2-3 & $8.06 \pm 0.25$ & $\begin{array}{c}1,2,4,5,6,8:^{* * * *} \\
7:^{* *}\end{array}$ \\
\hline C.MBT-Rvfs2-7 & $6.78 \pm 0.45$ & $1,5:^{* * * *}$ \\
& & $2,4,8:^{* * *}$ \\
& & $3,6:^{* *}$ \\
\hline \hline C.MBT-Rvfs2-1 & $4.13 \pm 0.18$ & \\
\hline C.MBT-Rvfs2-2 & $4.79 \pm 0.35$ & \\
\hline C.MBT-Rvfs2-4 & $4.33 \pm 0.31$ & \\
\hline C.MBT-Rvfs2-5 & $4.63 \pm 0.23$ & \\
\hline C.MBT-Rvfs2-6 & $4.67 \pm 0.21$ & \\
\hline C.MBT-Rvfs2-8 & $4.58 \pm 0.43$ & \\
\hline
\end{tabular}

$\S$ comparison of the survival time between the first group of strains (BALB/cByJ, C.MBT-Rvfs23 and C.MBT-Rvfs2-7) and all the others; strains of the second group did not statistically differ between them; numbers refer to C.MBT-Rvfs 2 substrains; ${ }^{* *}: \mathrm{p}<0.01 ;{ }^{* * *}$ : $<<0.001 ; * * * *$ : $\mathrm{p}<0.0001$.

\section{Table 1.}

$\S$ comparison of the survival time between the first group of strains (BALB/CByJ, C.MBTRvfs2-3 and C.MBT-Rvfs2-7) and all the others; strains of the second group did not statistically differ between them; numbers refer to C.MBT-Rvfs2 substrains; ${ }^{* *}: p<0.01$; $* * *: p<0.001 ; * * * *: p<0.0001$. 


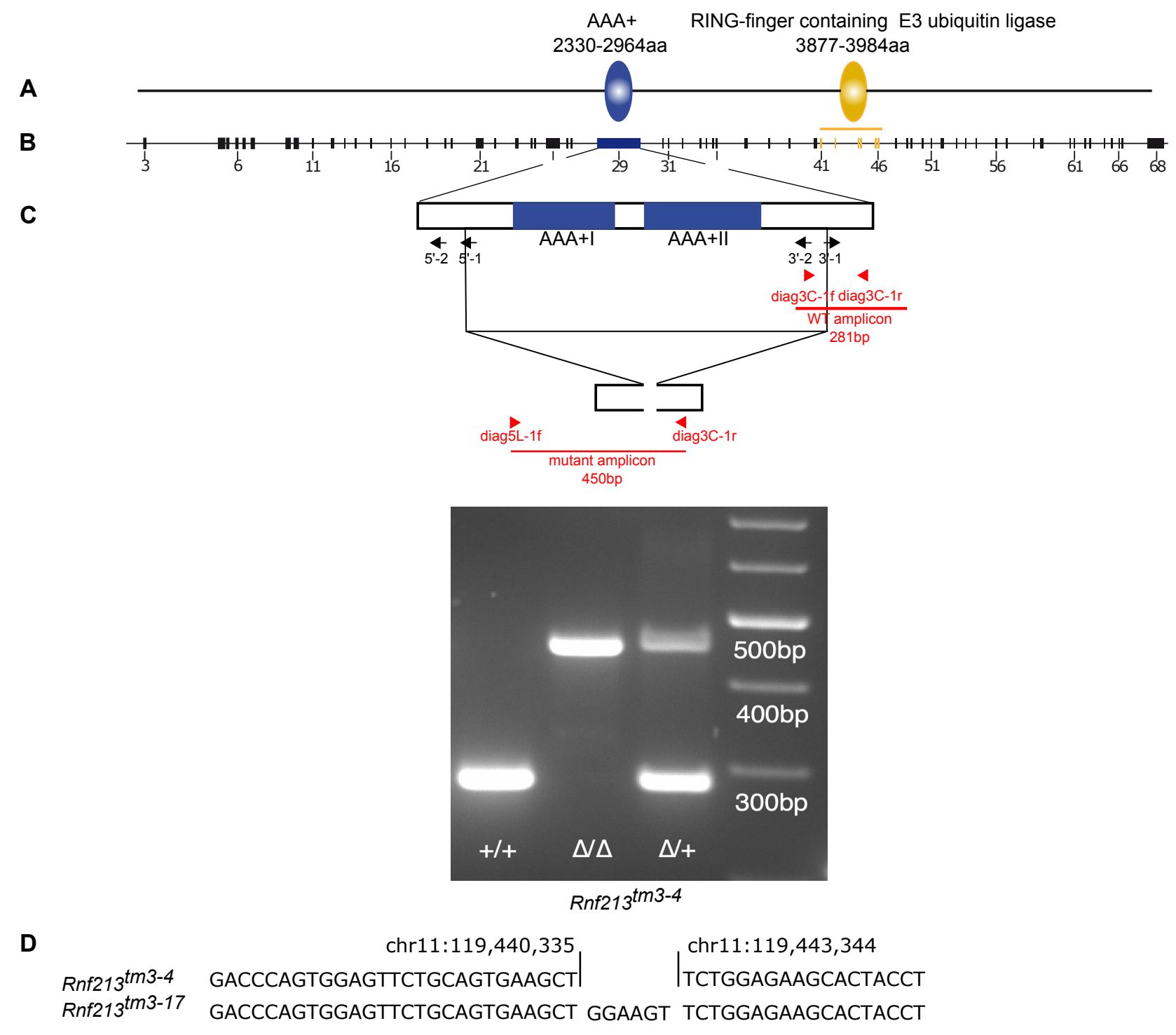

E

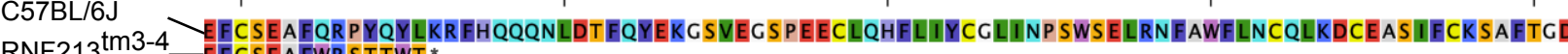

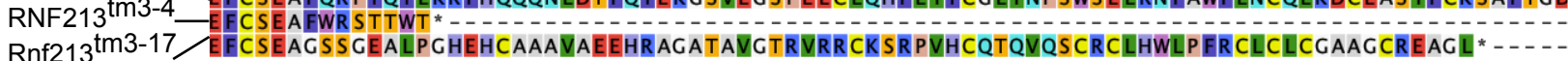

Figure 2.

Structure of the $R n f 213$ gene and the CRISP-Cas9-induced mutation. A: Organization of the RNF213 protein. The position of the RNF213 conserved domains is indicated. B: Structure of the endogenous mouse Rnf213 gene highlighting the position of exon 29 which encodes the AAA+ domains. C: The guides used to generate the deleted allele are shown as black arrows relative to the AAA+ domains. The primers used for genotyping transgenic mice are shown in red with the expected amplicon size. The expected deletion is shown as a triangle. PCR genotyping of $R n f 213^{+/+}$, Rnf $213^{t m 3 / t m 3}(\Delta / \Delta)$ and $R n f 213^{+/ t m 3}$ mice using primers: diag5L-1f, diag3C-1F and diag3C-1r . D: Genomic deletion in the two $R n f 213^{\operatorname{tm} 3}$ alleles analyzed. Rnf $213^{\text {tm } 3}$-17 carries a 6 base pair insertion within the deletion. E: Amino acid sequence of the C57BL/6J Rnf213 protein and of the truncated protein predicted from the deleted nucleotide sequence of the $R n f 213^{\operatorname{tm} 3 /}$ tm 3 -4 and Rnf213 $3^{\text {tm } 3 / t m 3}-17$ mutants. 


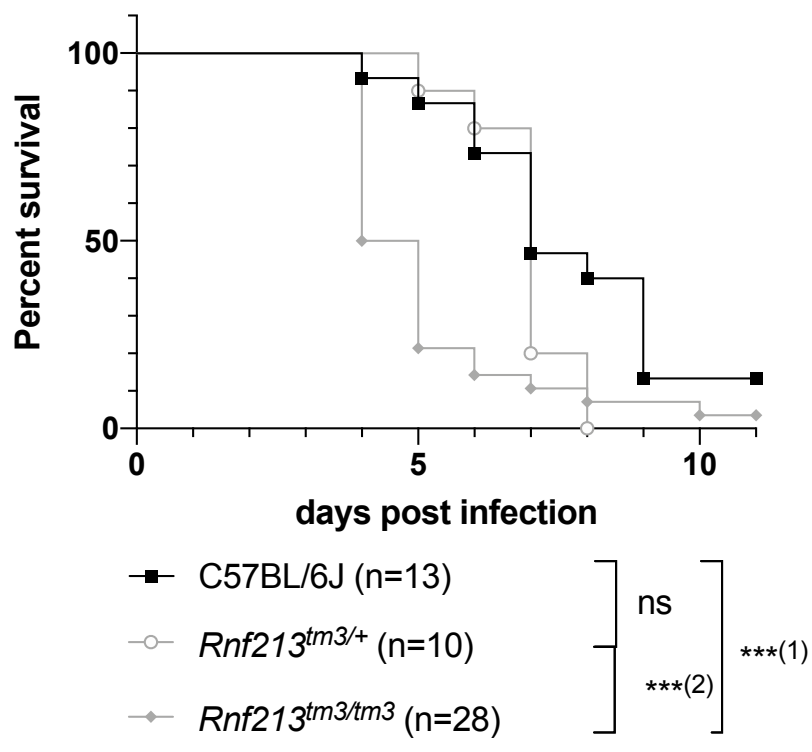

\section{Figure 3.}

Survival curves of $R n f 213^{t m 3 / t m 3}$, Rnf $213^{t m 3 /+}$, and C57BL/6J $\left(R n f 213^{+/+}\right)$male mice after infection with 100 PFU of RVFV ZH548 strain. Numbers of mice are given in brackets. Statistical test: Gehan-Breslow-

Wilcoxon test, $* * *(1) \mathrm{p}=0.0002, * * *(2) \mathrm{p}=0,0010$. 
Table S1. Markers used to build the physical map of the C.MBT-Rvfs2 subcongenic strains.

\begin{tabular}{|c|c|c|c|c|c|c|c|c|c|c|c|}
\hline Name & Position* & nfss $2-2$ & rufs2-3 & rvfs2-4 & nfs $2-5$ & rufs2-6 & rvfs2-7 & rvfs2-8 & Primer sequence & Amplicon & Band size \\
\hline slc26al1-1f & 119357215 & & & M & c & M & c & c & GCGGTCCTAGATTTTCCAACTGGGG & amplicon: 416pb -followed by restriction with NlaIII & BALB: $32 \mathrm{pb}+7 \mathrm{pb}+307 \mathrm{pb}+76 \mathrm{pt}$ \\
\hline slc26a11-1r & 119357630 & & & & & & & & TCTGAGTGTCTGGAACTGAACCCCG & & PWK: $32 \mathrm{pb}+7 \mathrm{pb}+365 \mathrm{pb}$ \\
\hline slc26al1-2f & 119380672 & & & & & & & & TCTAATCTGAGCTGAAGAGGTGGCC & PWK: 832 pb BALB: 841 & \\
\hline slc26al1-2r & 119381506 & & & & & & & & CGCCCATATACAGTGTTGGATGGGG & & \\
\hline Mir1932-2f & 119385695 & M & c & M & M & M & c & c & GGCTAGAATGTGTAAGTTTTGTAATTCTGG & BALB: 395, MBT: 210 & \\
\hline Mir1932-2r & 119386089 & & & & & & & & CCTTGGGGCAAATTTGATCTCCACC & & \\
\hline $\mathrm{mf} 213-2 \mathrm{f}$ & 119392890 & & c & & & M & & c & TTGGGGGAGTAGCTGGACTTGAGTTAGAGC & amplicon: 291pb -followed by restriction with NlaIII & BALB: $212 p b+22 p b+56 p b$ \\
\hline inf213-2r & 119393180 & & & & & & & & AGAAGGCAATTTCTGTCCGCACTCG & & PWK: $138 \mathrm{pb}+74 \mathrm{pb}+22 \mathrm{pb}+56 \mathrm{pb}$ \\
\hline Rnf213-exon00-1f & 119392806 & & & & & & & & CTTTTGAGCTTCCCTGGGCTTCG & amplicon: 900pb - sequenced & \\
\hline Rnf213-exon00-1r & 119393705 & & & & & & & c & GTGCTCTTCACTGCATAGGCCATCC & & C1 \\
\hline rnf213-1f & 119400214 & $\mathbf{M}$ & c & $\mathbf{M}$ & $\mathbf{M}$ & $\mathbf{M}$ & c & $\mathbf{M}$ & GACAGTAGGAATGGTTTTGGGAAGG & PWK: 545pb BALB: 739pb & \\
\hline inf213-1r & 119400952 & & & & & & & & CCCACTGCTGTAACAAATCTTGTCC & & \\
\hline EndoV-1f & 119528452 & & & & & & c & $\mathbf{M}$ & GCCAGTATTGAGTGTGGTTCCTCCC & PWK: $180 \mathrm{pb}$ BALB: $159 \mathrm{pb}$ & \\
\hline EndoV-1r & 119528631 & & & & & & & & GTCCACTCAGAACCTGTGAATGGGG & & \\
\hline Nptx1-1f & 119547995 & & & & & & c & M & CCGAAGACTACCAGAATACAGGGCC & amplicon: 636pb -followed by restriction with NlaIII & BALB: $172 \mathrm{pb}+102 \mathrm{pb}+361 \mathrm{pb}$ \\
\hline Nptx1-1r & 119548631 & & & & & & & & TTCAAGTGGACCGGTAAACCCAACG & & PWK: $172 \mathrm{pb}+463 \mathrm{pb}$ \\
\hline Gm11762-1f & 119570841 & & & & & & c & $\mathbf{M}$ & AGCAAGTGACCAGTTGACAGGAAGC & PWK: 419pb BALB: 297pb & \\
\hline Gm11762-1r & 119571260 & & & & & & & & CCTCACCATGGTCAGGGCTTTACC & & \\
\hline Rptor-1f & 119861491 & M & c & M & M & M & c & $\mathbf{M}$ & ATGGTCTCACTTCAAAGCCACTGGG & PWK: 248pb BALB: $\sim 300 \mathrm{pb}$ & \\
\hline Rptor-1r & 119861882 & & & & & & & & GAACGAACACAGGATCAGGGCTCTG & & \\
\hline Rptor-2f & 119899881 & & & & & & c & M & CTACCCACTGCGAATGTCTCCATCC & amplicon: $158 \mathrm{pb}$-followed by restriction with NlaIII & BALB: $117 \mathrm{pb}+16 \mathrm{pb}$ \\
\hline Rptor-2r & 119900038 & & & & & & & & ACCCTGACACCTGAGGTATCCTTCC & & PWK: $85 \mathrm{pb}+32 \mathrm{pb}+16 \mathrm{pb}$ \\
\hline Chmp6-2f & 119911581 & & & & & & c & & TTCCCTCCTTTCAGGTGACTCTGGC & PWK: 339pb BALB: 395pb & \\
\hline Chmp6-2r & 119911975 & & & & & & & & СTTCCCTTTGCCTCCCTTTGCTGG & & \\
\hline Chmp6-3f & 119917845 & & & & & & c & & CAGGGCCAGTCATCTGAGCAGG & amplicon: $248 \mathrm{pb}$-followed by restriction with NlaIII & BALB: $118+130$ \\
\hline Chmp6-3r & 119918092 & & & & & & & & GGGTATGTTCCTTAGACCAACACG & & PWK: 248 \\
\hline Chmp6-1f & 119927903 & & c & $\mathbf{M}$ & & $\mathbf{M}$ & M & & TGTGATTCCAAAGGAAAAGACAGTCC & PWK: 280 pb BALB $346 \mathrm{pb}$ & $\mathrm{C} 2$ \\
\hline Chmp6-1r & 119928248 & & & & & & & & TTTAAATTTGTAAACTGATGCCTTTCGC & & \\
\hline Baiap-1f & 120006436 & & & & & M & & M & CTCTGCATCGACACTCTGTCCCC & amplicon: $149 \mathrm{pb}$ followed by restriction with NlaIII & BALB: $51 \mathrm{pb}+97 \mathrm{pb}$ \\
\hline Baiap-1r & 120006584 & & & & & & & & CAGCCACCTCACCAGGAACACC & & PWK:51pb $+25 \mathrm{pb}+72 \mathrm{pb}$ \\
\hline Aatk-1f & 120014703 & M & c & M & $\mathbf{M}$ & c & M & $\mathbf{M}$ & CTACCCCAGGAGGACTGTGTCAGG & PWK: 127pb BALB: 180pb & \\
\hline Aatk-1r & 120014743 & & & & & & & & GTCCTCCCCAACAATATCCTGGTGC & & \\
\hline Azil-2f & 120064310 & & c & $\mathbf{M}$ & & c & & & AGTCGGAGCTAGTCCTGCAGTAAGG & amplicon: $820 \mathrm{pb}$ - sequenced & \\
\hline Azi1-2r & 120065129 & & & & & & & & TAGCCACCCAGAGTCTCCTTAGTCC & & \\
\hline Azi1-3f & 120085919 & & c & & & c & & M & CTAGGACTCCCTTGAGAGGACAGGG & amplicon: $161 \mathrm{pb}$-followed by restriction with NlallI & BALB: $92 \mathrm{pb}+65 \mathrm{pb}$ \\
\hline Azi1-3r & 120086079 & & & & & & & & GACCATGGTGAGTGTATGCTCCTGG & & PWK:157pb \\
\hline 11-rik120,1-1f & 120099574 & & M & & & c & & M & CATGACCCCTCCACTAGAGCTAGGG & amplicon :243pb -followed by restriction with NlallI & BALB: $243 \mathrm{pb}$ \\
\hline 11-rik120,1-1r & 120099816 & & & & & & & & TGGCCACCAACCACAAGAGTAAAGG & & PWK: $140 \mathrm{pb}+102 \mathrm{pb}$ \\
\hline slc38al0-1f & 120101727 & $\mathbf{M}$ & M & M & $\mathbf{M}$ & c & M & $\mathbf{M}$ & GGCTCTTACTATGACCCAGAGCAGC & PWK:198pb BALB: 379pb & \\
\hline slc38a10-1r & 120102105 & & & & & & & & CTTGAAGATGACACTGGCAGGTTGG & & \\
\hline Actg1-1f & 120341838 & M & M & c & $\mathbf{M}$ & c & M & M & CAGTGCTGGACTTGAAGGAGACTGG & PWK: 338pb, BALB: 725pb & \\
\hline Actg-1r & 120342562 & & & & & & & & TCTCTCCAGCCCTCTACCCCTTATT & & \\
\hline Nploc4-1f & 120424100 & M & M & c & M & c & M & M & CGCTTTGGTTAGGCCTAGTGGTTCA & PWK: 212pb, BALB: 409pb & \\
\hline Nploc4-1r & 120424508 & & & & & & & & ATGGACAGGGGACAAAACACGAGAC & & \\
\hline $\operatorname{ccdc137-1f}$ & 120460307 & M & M & c & $\mathbf{M}$ & c & M & M & TGGTGACTGTGTCCTCTGGTGATAG & PWK: 400 BALB: 586pb & \\
\hline ccdc137-1r & 120460892 & & & & & & & & TGTCCTGTTCTTAGACCCTCCAGTG & & \\
\hline P4hb-1f & 120568970 & $\mathbf{M}$ & M & c & $\mathbf{M}$ & c & M & $\mathbf{M}$ & ACAGCTCACTCAGAGAACCCAGAGC & PWK: 600 BALB: 400 & \\
\hline P4hb-1r & 120569573 & & & & & & & & CGGCACCAGTCAAAAATTTAAAGG & & \\
\hline Cbr2-1f & 120735744 & M & M & c & M & c & M & M & GGCCTTACAGCTGGATCTCATGAAGG & PWK: 368pb BALB: 776pb & \\
\hline Cbr2-1r & 120736519 & & & & & & & & GAGTTGGAGAAGTCACCCAATCCCG & & \\
\hline cd7-1f & 121043129 & M & M & c & M & c & M & M & AATGAGTTCACCTGGACCCACTAGC & MBT:150pb BALB: 300pb & \\
\hline cd7-1r & 121043421 & & & & & & & & CTAGACGTGGACTAGGAAGCCCAAT & & \\
\hline Sectmla-1f & 121095593 & M & M & c & M & c & M & M & TCAGGCAAGGGTTGTGAAAGTTGGG & MBT: $<<300 p b$ BALB: - & \\
\hline Sectmla-1r & 121096410 & & & & & & & & ATGACTTTAGTCCCAGCCCTCCAGA & & \\
\hline Tex19.2-1f & 121126146 & M & M & c & $\mathbf{M}$ & c & & & TCAGGACTCTTCACAGGTTGCAGAC & MBT: 1.2kb PWK: 214 BALB: 1437bp & \\
\hline Tex19.2-1r & 121127582 & & & & & & & & GGGTTACCACTTTCTGCTCAGGGTT & & \\
\hline Tex19.1-1f & 121152273 & $\mathbf{M}$ & M & c & $\mathbf{M}$ & c & & & ACAAGCCAAGCTCTGTCTCCATCAC & MBT: 550pb PWK: 441pb BALB: 585pb & \\
\hline Tex19.1-1r & 121152857 & & & & & & & & CCCAACCGTTGTGTTAGAACCTGGT & & \\
\hline Hexdc -1f & 121217621 & c & c & c & $\mathbf{M}$ & c & M & $\mathbf{M}$ & CCCAAAACACAGAAACACAGCTGCA & PWK: 367pb BALB: 549pb & \\
\hline Hexdc -1r & 121218169 & & & & & & & & CCGTACTGACCTTTGCCATGGACAT & & \\
\hline Narf-1f & 121247120 & c & c & c & M & c & M & M & TCAGTGGCCTAAAGTGAAGAAGAAGG & PWK: 230 BALB: 486 & \\
\hline Narf-1r & 121247605 & & & & & & & & ACATATGGCTTCCTCCCTAAGATGG & & \\
\hline Foxk2-1f & 121323858 & c & c & c & M & c & M & M & GCAGCAGTGTTCTGGGTTAACAGGA & PWK: 593pb BALB: 796pb & \\
\hline Foxk2-1r & 121324653 & & & & & & & & GTCCTTTCAGCAGTCTGCCTCAGTT & & \\
\hline wdr45b-1f & 121344547 & c & c & c & M & c & M & M & GCAAGGTGGGCCAAACCAAGGTAAT & PWK: 315pb BALB: 506pb & \\
\hline wdr45b-1r & 121345052 & & & & & & & & GGCAGAAAAGGCCCGACTCACTTAA & & \\
\hline B3gntl1-1f & 121649133 & c & c & c & $\mathbf{M}$ & c & M & M & CCTCTCGGTCTGGTTGTTGGTGATC & PWK: 198pb BALB: 690pb & \\
\hline B3gntl1-1r & 121649822 & & & & & & & & СТCCСТCACTAAGACAAGGCCCTA & & \\
\hline Metrnl-1f & 121711717 & c & c & c & $\mathbf{M}$ & c & $\mathbf{M}$ & M & TCATTGAAGAAGCCCAGGCCTTAGC & PWK: 228pb BALB: 674pb & \\
\hline Metrnl-1r & 121712390 & & & & & & & & ACTACCTCCTTCAGTTGTGCTTGGC & & \\
\hline
\end{tabular}

* according to GRCm38-mm10 
Table S2. Nucleotide and protein variants between the BALB/cJ and the PWK/PhJ strains in the $R$ nf213 gene (Chr: 11:119,393,100-119,487,418).

\begin{tabular}{|c|c|c|c|c|c|}
\hline \multicolumn{3}{|c|}{ Nucleotide variant* } & \multirow[t]{2}{*}{ Functional impact } & \multicolumn{2}{|c|}{ Protein variant** } \\
\hline Position & BALB & PWK & & Position & Region \\
\hline \multicolumn{6}{|l|}{ SNPs } \\
\hline $119,402,554$ & $\mathrm{~T}$ & $\mathrm{C}$ & missense_variant & S44P & exon03 \\
\hline $119,402,617$ & $\mathrm{C}$ & $\mathrm{T}$ & missense_variant & L65S & exon03 \\
\hline $119,402,618$ & $\mathrm{~T}$ & $\mathrm{C}$ & missense_variant & L65S & exon03 \\
\hline $119,402,668$ & A & G & missense_variant & N82D & exon03 \\
\hline $119,408,883$ & $\mathrm{C}$ & $\mathrm{T}$ & missense_variant & P146L & exon04 \\
\hline $119,408,898$ & $\mathrm{~T}$ & $\mathrm{~A}$ & missense_variant & $\mathrm{I} 151 \mathrm{~N}$ & exon04 \\
\hline $119,410,735$ & $\mathrm{C}$ & $\mathrm{T}$ & missense_variant & R345W & exon07 \\
\hline $119,414,325$ & $\mathrm{~T}$ & $\mathrm{C}$ & splice_region_variant & 463 & exon08/09 \\
\hline $119,414,329$ & $\mathrm{C}$ & $\mathrm{T}$ & splice_region_variant & 463 & exon08/09 \\
\hline $119,414,379$ & $\mathrm{~T}$ & $\mathrm{C}$ & missense_variant & F479L & exon09 \\
\hline 119414400 & $\mathrm{~T}$ & A & missense_variant & F86I & exon09 \\
\hline $119,414,423$ & $\mathrm{C}$ & A & missense_variant & D493E & exon09 \\
\hline $119,415,046$ & $\mathrm{~T}$ & $\mathrm{C}$ & missense_variant & M560T & exon10 \\
\hline $119,415,199$ & $\mathrm{G}$ & $\mathrm{A}$ & missense_variant & R611K & exon 10 \\
\hline $119,415,214$ & $\mathrm{~T}$ & $\mathrm{C}$ & missense_variant & L616S & exon10 \\
\hline $119,415,286$ & $\mathrm{C}$ & G & missense_variant & P640R & exon10 \\
\hline $119,416,642$ & $\mathrm{~T}$ & G & $\begin{array}{l}\text { splice_region_variant } \\
\text { synonymous_variant }\end{array}$ & 709 & exon $11 / 12$ \\
\hline $119,418,153$ & A & G & missense_variant & M736V & exon12 \\
\hline $119,420,089$ & A & $\mathrm{T}$ & missense_variant & Q811L & exon14 \\
\hline $119,420,101$ & $\mathrm{~T}$ & $\mathrm{C}$ & missense_variant & $\mathrm{I} 815 \mathrm{~T}$ & exon14 \\
\hline $119,421,309$ & $\mathrm{~T}$ & $\mathrm{C}$ & splice_region_variant & 856 & exon $14 / 15$ \\
\hline $119,423,029$ & G & A & missense_variant & G918S & exon16 \\
\hline $119,424,276$ & $\mathrm{C}$ & A & $\begin{array}{c}\text { missense_variant } \\
\text { splice_region_variant }\end{array}$ & Q982K & exon $17 / 18$ \\
\hline $119,427,672$ & G & A & splice_region_variant & 1098 & exon $19 / 20$ \\
\hline $119,428,055$ & G & A & missense_variant & $\mathrm{R} 1113 \mathrm{~K}$ & exon 20 \\
\hline $119,430,298$ & $\mathrm{~T}$ & G & missense_variant & L1193I & exon 21 \\
\hline $119,430,520$ & $\mathrm{~T}$ & $\mathrm{C}$ & missense_variant & V1267A & exon 21 \\
\hline $119,430,606$ & $\mathrm{C}$ & G & missense_variant & R1296G & exon 21 \\
\hline $119,431,730$ & $\mathrm{C}$ & G & missense_variant & A1391G & exon 22 \\
\hline $119,435,905$ & $\mathrm{C}$ & $\mathrm{T}$ & splice_region_variant & 1575 & exon $25 / 26$ \\
\hline $119,436,016$ & A & G & missense_variant & R1610G & exon 26 \\
\hline $119,436,068$ & $\mathrm{G}$ & A & missense_variant & K1627S & exon 26 \\
\hline $119,436,069$ & $\mathrm{~T}$ & $\mathrm{G}$ & missense_variant & K1627S & exon 26 \\
\hline $119,436,308$ & $\mathrm{G}$ & A & missense_variant & $\mathrm{S} 1707 \mathrm{~N}$ & exon 26 \\
\hline $119,436,397$ & A & $\mathrm{G}$ & missense_variant & $\mathrm{S} 2052 \mathrm{G}$ & exon 26 \\
\hline $119,437,613$ & $\mathrm{C}$ & $\mathrm{T}$ & splice_region_variant & 1957 & exon $26 / 27$ \\
\hline $119,437,800$ & $\mathrm{~A}$ & G & missense_variant & I2920V & exon 27 \\
\hline $119,438,060$ & $\mathrm{~T}$ & $\mathrm{C}$ & missense_variant & S2052P & exon 28 \\
\hline $119,440,315$ & G & A & missense_variant & $\mathrm{V} 2117 \mathrm{M}$ & exon 29 \\
\hline $119,440,407$ & A & $\mathrm{C}$ & missense_variant & $\mathrm{K} 2147 \mathrm{~N}$ & exon29 \\
\hline $119,440,554$ & G & $\mathrm{T}$ & missense_variant & $\mathrm{K} 2196 \mathrm{~N}$ & exon29 \\
\hline $119,441,657$ & $\mathrm{C}$ & $\mathrm{T}$ & missense_variant & $\mathrm{S} 2564 \mathrm{~F}$ & exon 29 \\
\hline $119,441,675$ & A & G & missense_variant & N2570S & exon 29 \\
\hline $119,446,209$ & G & $\mathrm{T}$ & splice_region_variant & 3352 & exon $31 / 32$ \\
\hline $119,449,331$ & A & G & missense_variant & T3462A & exon34 \\
\hline $119,449,812$ & $\mathrm{~T}$ & $\mathrm{C}$ & missense_variant & V3499A & exon35 \\
\hline
\end{tabular}




\begin{tabular}{|c|c|c|c|c|c|}
\hline $119,460,010$ & $\mathrm{~T}$ & G & missense_variant & H3889Q & exon42 \\
\hline $119,467,227$ & A & $\mathrm{C}$ & $\begin{array}{l}\text { missense_variant } \\
\text { splice_region_variant }\end{array}$ & 4268 & exon $49 / 50$ \\
\hline $119,479,673$ & A & G & splice_region_variant & 4765 & exon60/61 \\
\hline $119,479,867$ & $\mathrm{G}$ & $\mathrm{C}$ & missense_variant & G4766A & exon61 \\
\hline $119,480,890$ & $\mathrm{C}$ & $\mathrm{A}$ & missense_variant & N4839K & exon62 \\
\hline \multicolumn{6}{|l|}{ Indels } \\
\hline $119,409,459$ & $\mathrm{C}$ & CAGG & in frame & $-262 G$ & exon05 \\
\hline $119,440,701$ & TGAA & $\mathrm{T}$ & in frame & E2246- & exon 29 \\
\hline $119,449,874$ & $\mathrm{G}$ & GGTAA & splice_region_variant & 3519 & exon $35 / 36$ \\
\hline $119,467,356$ & $\mathrm{C}$ & $\mathrm{CG}$ & splice_region_variant & 4307 & exon $50 / 51$ \\
\hline
\end{tabular}

* from the Sanger Institute Mouse Genomes Project

(https://www.sanger.ac.uk/sanger/Mouse_SnpViewer/)

** according to the A0A171EBL2 sequence (https://www.uniprot.org/uniprot/A0A171EBL2) 